\title{
GRAB-N-PULL: AN OPTIMIZATION FRAMEWORK FOR FAIRNESS-ACHIEVING NETWORKS
}

\author{
Mojtaba Soltanalian ${ }^{*+}$, Ahmad Gharanjik ${ }^{\dagger}$, M. R. Bhavani Shankar ${ }^{\dagger}$ and Björn Ottersten ${ }^{\dagger}$ \\ ${ }^{+}$Dept. of Electrical and Computer Engineering, University of Illinois at Chicago \\ ${ }^{\dagger}$ Interdisciplinary Centre for Security, Reliability and Trust (SnT), University of Luxembourg
}

\begin{abstract}
In this paper, we present an optimization framework for designing precoding (a.k.a. beamforming) signals that are instrumental in achieving a fair user performance through the networks. The precoding design problem in such scenarios can typically be formulated as a non-convex max-min fractional quadratic program. Using a penalized version of the original design problem, we derive a simplified quadratic reformulation of the problem in terms of the signal (to be designed). Each iteration of the proposed design framework consists of a combination of power method-like iterations and the Gram-Schmidt process, and as a result, enjoys a low computational cost. Moreover, the suggested approach can handle various types of signal constraints such as total-power, per-antenna power, unimodularity, or discrete-phase requirements - an advantage which is not shared by other existing approaches in the literature.
\end{abstract}

Index Terms - Beamforming, fractional programming, maxmin fairness, non-convex optimization, precoding

\section{INTRODUCTION}

A widely used proactive interpretation of fairness in wireless networks, referred to as the max-min fairness [1-8], is to allocate the available resources in order to maximize the minimal user performance in the network. In such scenarios, a judicious design of the precoding signals for different users can be viewed as a vital part of the network configuration.

\subsection{Problem Formulation}

We consider the precoding problem for a downlink channel, with an $M$-antenna transmitter and $K$ single-antenna users. Let $\boldsymbol{h}_{i} \in \mathbb{C}^{M}$ denote the channel between the transmit antennas and the $i^{\text {th }}$ user. Also let $\boldsymbol{w}_{i} \in \mathbb{C}^{M}$ denote the precoding vector corresponding to the $i^{\text {th }}$ user. To form the data stream to the users, any complex symbol to be transmitted, will be modulated by the precoding vector of the intended user. The precoding vectors are to be designed in order to enhance the network performance. In particular, the signal-to-noiseplus-interference ratio (SINR) of the $i^{\text {th }}$ user is given by [9]

$$
\operatorname{SINR}_{i}=\frac{\boldsymbol{w}_{i}^{H} \boldsymbol{R}_{i} \boldsymbol{w}_{i}}{\left(\sum_{j \in[K] \backslash\{i\}} \boldsymbol{w}_{j}^{H} \boldsymbol{R}_{i} \boldsymbol{w}_{j}\right)+\sigma_{i}^{2}}, \quad \forall i \in[K],
$$

where $\boldsymbol{R}_{i}=\mathbb{E}\left\{\boldsymbol{h}_{i} \boldsymbol{h}_{i}^{H}\right\}$ is the covariance matrix of the $i^{\text {th }}$ channel, $\sigma_{i}^{2}$ denotes the variance of the zero-mean additive white Gaussian

This work was supported in part by the National Research Fund (FNR), Luxembourg under AFR grant (Reference 5779106).

*Corresponding author-email: msol@uic.edu noise (AWGN), and $[K]=\{1,2, \cdots, K\}$. Note that by a specific reformulation, the SINR metric in (1) can be rewritten as a fractional quadratic criterion. To see this, define the stacked precoding vector $\boldsymbol{w} \in \mathbb{C}^{N}$ (with $N=K M$ ) as

$$
\boldsymbol{w} \triangleq \operatorname{vec}\left(\left[\boldsymbol{w}_{1} \boldsymbol{w}_{2} \cdots \boldsymbol{w}_{K}\right]\right),
$$

and observe that (1) will increase for any increased scaling of $\boldsymbol{w}$. As a result, any finite-energy constraint on $\boldsymbol{w}$ while maximizing $\left\{\mathrm{SINR}_{i}\right\}$ will be active, i.e. it will be satisfied with equality. Accordingly, suppose $\|\boldsymbol{w}\|_{2}^{2}=P$, and let

$$
\begin{array}{ll}
\boldsymbol{A}_{i} \triangleq \boldsymbol{R}_{i} \otimes \operatorname{Diag}\left(\boldsymbol{e}_{i}\right), & \forall i \in[K], \\
\boldsymbol{B}_{i} \triangleq \boldsymbol{R}_{i} \otimes\left(\boldsymbol{I}_{K}-\operatorname{Diag}\left(\boldsymbol{e}_{i}\right)\right)+\frac{\sigma_{i}^{2}}{P} \boldsymbol{I}_{N}, & \forall i \in[K],
\end{array}
$$

where Diag (.) denotes the diagonal matrix formed by the entries of the vector argument, $\otimes$ stands for the Kronecker product of matrices, and $\boldsymbol{e}_{i}$ is the the $i^{\text {th }}$ standard basis vector in $\mathbb{C}^{K}$. Now, it is not difficult to verify that

$$
\mathrm{SINR}_{i}=\frac{\boldsymbol{w}^{H} \boldsymbol{A}_{i} \boldsymbol{w}}{\boldsymbol{w}^{H} \boldsymbol{B}_{i} \boldsymbol{w}}, \quad \forall i \in[K],
$$

in which $\left\{\boldsymbol{A}_{i}\right\}$ are positive semidefinite (PSD) and $\left\{\boldsymbol{B}_{i}\right\}$ are positive definite (PD). Finally, the precoding design problem for maximizing the minimal user SINR performance can be formulated as

$$
\begin{aligned}
\mathcal{P}_{1}: & \max _{\boldsymbol{w}} \quad \min _{i \in[K]}\left\{\frac{\boldsymbol{w}^{H} \boldsymbol{A}_{i} \boldsymbol{w}}{\boldsymbol{w}^{H} \boldsymbol{B}_{i} \boldsymbol{w}}\right\} \\
\text { s. t. } & \boldsymbol{w} \in \Omega,
\end{aligned}
$$

where $\boldsymbol{\Omega}$ denotes the feasible set of $\boldsymbol{w}$ determined by the associated signal constraints. Note that $\mathcal{P}_{1}$ may also be used to formulate the weighted SINR optimization problems; see [1,2] for details.

\subsection{Contributions of this Work}

The above precoding design problem has been studied extensively in the literature when $\Omega$ denotes a total-power or per-antenna power constraint (see e.g., [1-8] and the references therein). As a result, different approaches have been proposed to solve the design problem, including those based on uplink-downlink duality [1], the Lagrangian duality [3] and quasi-convex formulations [8].

In this work, we propose a novel optimization framework (which we call Grab-n-Pull) that can efficiently tackle $\mathcal{P}_{1}$. Note that the proposed framework subsumes the traditional total-power or perantenna power constraints, while also allowing for intricate signal constraints such as unimodularity or discrete-phase requirements.

Remark 1: It is worth mentioning that the problem formulation $\mathcal{P}_{1}$ in (6) is also useful in other applications, including e.g., waveform design for radar [10,11], and relay beamforming [12,13]. 


\section{THE PROPOSED FRAMEWORK}

We begin by considering a reformulated version of $\mathcal{P}_{1}$; namely,

$$
\begin{aligned}
& \mathcal{P}_{2}: \max _{\boldsymbol{w}} \quad \min _{i \in[K]}\left\{\lambda_{i}\right\} \\
& \text { s. t. } \boldsymbol{w} \in \Omega, \\
& \lambda_{i}=\frac{\boldsymbol{w}^{H} \boldsymbol{A}_{i} \boldsymbol{w}}{\boldsymbol{w}^{H} \boldsymbol{B}_{i} \boldsymbol{w}}, \forall i \in[K] .
\end{aligned}
$$

Note that (8) holds if and only if $\left\|\boldsymbol{A}_{i}^{\frac{1}{2}} \boldsymbol{w}\right\|_{2}^{2}=\lambda_{i}\left\|\boldsymbol{B}_{i}^{\frac{1}{2}} \boldsymbol{w}\right\|_{2}^{2}$, or equivalently $\left\|\boldsymbol{A}_{i}^{\frac{1}{2}} \boldsymbol{w}\right\|_{2}=\sqrt{\lambda_{i}}\left\|\boldsymbol{B}_{i}^{\frac{1}{2}} \boldsymbol{w}\right\|_{2}$, where (.) $)^{\frac{1}{2}}$ denotes the Hermitian square-root of the matrix argument [14]. In particular, the lefthand side of (8) is close to the right-hand side of (8) if and only if $\left\|\boldsymbol{A}_{i}^{\frac{1}{2}} \boldsymbol{w}\right\|_{2}$ is close to $\sqrt{\lambda_{i}}\left\|\boldsymbol{B}_{i}^{\frac{1}{2}} \boldsymbol{w}\right\|_{2}$. Therefore, by employing the auxiliary variables $\left\{\lambda_{i}\right\}$, one can consider the following optimization problem as an alternative to $\mathcal{P}_{2}$ (and $\mathcal{P}_{1}$ ):

$$
\begin{array}{cl}
\mathcal{P}_{3}: & \max _{\boldsymbol{w},\left\{\lambda_{i}\right\}} \min _{i \in[K]}\left\{\lambda_{i}\right\}-\eta \sum_{i=1}^{K}\left(\left\|\boldsymbol{A}_{i}^{\frac{1}{2}} \boldsymbol{w}\right\|_{2}-\sqrt{\lambda_{i}}\left\|\boldsymbol{B}_{i}^{\frac{1}{2}} \boldsymbol{w}\right\|_{2}\right)^{2} \\
\text { s. t. } & \boldsymbol{w} \in \Omega ; \lambda_{i} \geq 0, \forall i \in[K]
\end{array}
$$

in which $\eta>0$ determines the weight of the penalty-term added to the original objective of $\mathcal{P}_{2}$; and where $\mathcal{P}_{3}$ and $\mathcal{P}_{2}$ coincide as $\eta \rightarrow+\infty$. Note that optimizing $\mathcal{P}_{3}$ with respect to (w. r. t.) $\boldsymbol{w}$ may require rewriting $\mathcal{P}_{3}$ as a quartic objective in $\boldsymbol{w}$. To circumvent this, we continue by introducing $\mathcal{P}_{4}$-yet another alternative objective:

$$
\begin{gathered}
\mathcal{P}_{4}: \max _{\boldsymbol{w},\left\{\lambda_{i}\right\},\left\{\boldsymbol{Q}_{i}\right\}} \min _{i \in[K]}\left\{\lambda_{i}\right\}-\eta \sum_{i=1}^{K}\left\|\boldsymbol{A}_{i}^{\frac{1}{2}} \boldsymbol{w}-\sqrt{\lambda_{i}} \boldsymbol{Q}_{i} \boldsymbol{B}_{i}^{\frac{1}{2}} \boldsymbol{w}\right\|_{2}^{2} \\
\text { s. t. } \quad \boldsymbol{w} \in \Omega, \lambda_{i} \geq 0, \forall i \in[K] ; \\
\boldsymbol{Q}_{i}^{H} \boldsymbol{Q}_{i}=\boldsymbol{I}_{N}, \forall i \in[K] .
\end{gathered}
$$

Towards understanding the equivalence of $\mathcal{P}_{3}$ and $\mathcal{P}_{4}$, observe that the maximizer $\boldsymbol{Q}_{i}$ of $\mathcal{P}_{4}$ is a unitary rotation matrix that aligns the vector $\boldsymbol{B}_{i}^{\frac{1}{2}} \boldsymbol{w}$ in the same direction as $\boldsymbol{A}_{i}^{\frac{1}{2}} \boldsymbol{w}$, without changing its $\ell_{2}$-norm (i.e. $\left\|\boldsymbol{B}_{i}^{\frac{1}{2}} \boldsymbol{w}\right\|_{2}$ ). More precisely, at the maximizer $\boldsymbol{Q}_{i}$ of $\mathcal{P}_{4}$, we have that

$$
\boldsymbol{Q}_{i} \boldsymbol{B}_{i}^{\frac{1}{2}} \boldsymbol{w}=\left(\frac{\left\|\boldsymbol{B}_{i}^{\frac{1}{2}} \boldsymbol{w}\right\|_{2}}{\left\|\boldsymbol{A}_{i}^{\frac{1}{2}} \boldsymbol{w}\right\|_{2}}\right) \boldsymbol{A}_{i}^{\frac{1}{2}} \boldsymbol{w}
$$

In contrast to $\mathcal{P}_{3}$, the optimization problem $\mathcal{P}_{4}$ can be easily rewritten as a quadratic program $(\mathrm{QP})$ in $\boldsymbol{w}$; a widely studied type of program that facilitates the usage of power method-like iterations, and thus employing different signal constraints $\Omega$-more on this later. In the following, we propose an efficient iterative optimization framework based on a separate optimization of the objective of $\mathcal{P}_{4}$ over its three partition of variables at each iteration, viz. $\boldsymbol{w},\left\{\boldsymbol{Q}_{i}\right\}$, and $\left\{\lambda_{i}\right\}$, where the iterations can be started from any arbitrary initialization.

\subsection{Power Method-Like Iterations (Optimization w. r. t. $w$ )}

For fixed $\left\{\boldsymbol{Q}_{i}\right\}$ and $\left\{\lambda_{i}\right\}$, one can optimize $\mathcal{P}_{4}$ w. r. t. $\boldsymbol{w}$ via minimizing the criterion:

$$
\sum_{i=1}^{K}\left\|\boldsymbol{A}_{i}^{\frac{1}{2}} \boldsymbol{w}-\sqrt{\lambda_{i}} \boldsymbol{Q}_{i} \boldsymbol{B}_{i}^{\frac{1}{2}} \boldsymbol{w}\right\|_{2}^{2}=\boldsymbol{w}^{H} \boldsymbol{R} \boldsymbol{w}
$$

where

$$
\boldsymbol{R}=\sum_{i=1}^{K}\left\{\left(\boldsymbol{A}_{i}+\lambda_{i} \boldsymbol{B}_{i}\right)-\sqrt{\lambda_{i}}\left(\boldsymbol{A}_{i}^{\frac{1}{2}} \boldsymbol{Q}_{i} \boldsymbol{B}_{i}^{\frac{1}{2}}+\boldsymbol{B}_{i}^{\frac{1}{2}} \boldsymbol{Q}_{i}^{H} \boldsymbol{A}_{i}^{\frac{1}{2}}\right)\right\}
$$

Due to the fact that $\Omega$ enforces a fixed $\ell_{2}$-norm on $\boldsymbol{w}$ (i.e. $\|\boldsymbol{w}\|_{2}^{2}=$ $P$ ), by defining the PD matrix $\hat{\boldsymbol{R}} \triangleq \mu \boldsymbol{I}-\boldsymbol{R}$ (with $\mu>0$ being larger than the maximum eigenvalue of $\boldsymbol{R}$ ), we have that $\boldsymbol{w}^{H} \boldsymbol{R} \boldsymbol{w}=$ $-\boldsymbol{w}^{H} \hat{\boldsymbol{R}} \boldsymbol{w}+\mu P$ in which $\mu P$ is constant. Consequently, one can minimize (or decrease monotonically) the criterion in (13) by maximizing (or increasing monotonically) the objective of the following optimization problem:

$$
\begin{array}{cc}
\max _{\boldsymbol{w}} . & \boldsymbol{w}^{H} \hat{\boldsymbol{R}} \boldsymbol{w} \\
\text { s. t. } & \boldsymbol{w} \in \Omega .
\end{array}
$$

Although (14) is NP-hard for a general signal constraint set $[15,16]$, a monotonically increasing objective of (14) can be obtained using power method-like iterations developed in [16], and [17]; namely, we update $\boldsymbol{w}$ iteratively by solving the following nearest-vector problem at each iteration:

$$
\begin{array}{cl}
\min _{\boldsymbol{w}^{(s+1)}} & \left\|\boldsymbol{w}^{(s+1)}-\hat{\boldsymbol{R}} \boldsymbol{w}^{(s)}\right\|_{2} \\
\text { s. t. } & \boldsymbol{w}^{(s+1)} \in \Omega,
\end{array}
$$

where $s$ denotes the internal iteration number, and $\boldsymbol{w}^{(0)}$ is the current value of $\boldsymbol{w}$. Note that we can continue updating $\boldsymbol{w}$ until convergence in the objective of (14), or for a fixed number of steps, say $S$.

Now, we take a deeper look at various signal constraints $\Omega$ typically used in practice, as well as their associated constrained solutions to (15):

- Total-power constraint: In this case, $\Omega$ can be defined as $\Omega=\left\{\boldsymbol{w}:\|\boldsymbol{w}\|_{2}^{2}=P\right\}$, where $P>0$. Then, the set of power method-like iterations in (15) boils down to a typical power method aiming to find the dominant eigenvector of $\hat{\boldsymbol{R}}$, however with an additional scaling to attain a power of $P$.

- Per-antenna power constraint: We consider $M$ antennas with assigned power values $\left\{P_{i}\right\}$, and assume that $K=N / M$ entries of $\boldsymbol{w}$ are devoted to each antenna. As a result, we can solve (15) by considering the nearest-vector problem for sub-vectors associated with each antenna separatelyi.e., $M$ nearest-vector problems all with vector arguments of length $K$.

- Unimodular signal design: The set of unimodular codes is defined as $\Omega=\left\{e^{j \varphi}: \varphi \in[0,2 \pi)\right\}^{N}$. The unimodular solution to (15) is simply given by $(P=N)$ :

$$
\boldsymbol{w}^{(s+1)}=\exp \left(j \arg \left(\hat{\boldsymbol{R}} \boldsymbol{w}^{(s)}\right)\right) .
$$

- Discrete-phase signal design: We define the set of discretephase signals as, $\Omega=\left\{e^{j \frac{2 \pi}{Q} q}: q=0,1, \cdots, Q-1\right\}^{N}$ where $Q$ denotes the phase quantization level. The discretephase solution to (15) is given by $(P=N)$ :

$$
\boldsymbol{w}^{(s+1)}=\exp \left(j \mu_{Q}\left(\arg \left(\hat{\boldsymbol{R}} \boldsymbol{w}^{(s)}\right)\right)\right)
$$

where $\mu_{Q}($.$) yields (for each entry of the vector argument)$ the closest element in the $Q$-ary alphabet.

Finally, we refer the interested reader to find more details on the properties of power method-like iterations in [16]- [18]. 


\subsection{Rotation-Aided Fitting (Optimization w. r. t. $\left\{\boldsymbol{Q}_{i}\right\}$ )}

Suppose $\boldsymbol{w}$ and $\left\{\lambda_{i}\right\}$ are fixed. As discussed earlier, the maximizer $\boldsymbol{Q}_{i}$ of $\mathcal{P}_{4}$ is a rotation matrix that maps $\boldsymbol{B}_{i}^{\frac{1}{2}} \boldsymbol{w}$ in the same direction as $\boldsymbol{A}_{i}^{\frac{1}{2}} \boldsymbol{w}$. Let

$$
\left\{\begin{array}{l}
\boldsymbol{u}_{i}=\boldsymbol{A}_{i}^{\frac{1}{2}} \boldsymbol{w} /\left\|\boldsymbol{A}_{i}^{\frac{1}{2}} \boldsymbol{w}\right\|_{2} \\
\boldsymbol{v}_{i}=\boldsymbol{B}_{i}^{\frac{1}{2}} \boldsymbol{w} /\left\|\boldsymbol{B}_{i}^{\frac{1}{2}} \boldsymbol{w}\right\|_{2}
\end{array}\right.
$$

and note that (12) can be rewritten as $\boldsymbol{u}_{i}=\boldsymbol{Q}_{i} \boldsymbol{v}_{i}$ for all $i \in[K]$. We define the unitary matrices $\boldsymbol{Q}_{\boldsymbol{u}_{i}}$ and $\boldsymbol{Q}_{\boldsymbol{v}_{i}}$ in $\mathbb{C}^{N \times N}$ as

$$
\begin{aligned}
& \boldsymbol{Q}_{\boldsymbol{u}_{i}}=\left[\widehat{\boldsymbol{u}}_{i 1}, \widehat{\boldsymbol{u}}_{i 2}, \ldots, \widehat{\boldsymbol{u}}_{i N}\right] \\
& \boldsymbol{Q}_{\boldsymbol{v}_{i}}=\left[\widehat{\boldsymbol{v}}_{i 1}, \widehat{\boldsymbol{v}}_{i 2}, \ldots, \widehat{\boldsymbol{v}}_{i N}\right]
\end{aligned}
$$

where each of the sets $\left\{\widehat{\boldsymbol{u}}_{i j}\right\}_{j}$ and $\left\{\widehat{\boldsymbol{v}}_{i j}\right\}_{j}, j \in[N]$, builds an orthonormal basis for $\mathbb{C}^{N}$, and $\widehat{\boldsymbol{u}}_{i 1}=\boldsymbol{u}_{i}, \widehat{\boldsymbol{v}}_{i 1}=\boldsymbol{v}_{i}$. Based on the above definitions, the following lemma constructs the optimal $\boldsymbol{Q}_{i}$ (the proof of Lemma 1 is straightforward and omitted herein):

Lemma 1. The maximizer $\left\{\boldsymbol{Q}_{i}\right\}$ of $\mathcal{P}_{4}$ can be found as $\boldsymbol{Q}_{i}=$ $\boldsymbol{Q}_{\boldsymbol{u}_{i}} \boldsymbol{Q}_{\boldsymbol{v}_{i}}^{H}$ for all $i \in[K]$.

Remark 2: Note that $\left\{\boldsymbol{Q}_{\boldsymbol{u}_{i}}\right\}$ and $\left\{\boldsymbol{Q}_{\boldsymbol{v}_{i}}\right\}$ can be obtained via the Gram-Schmidt process, and are not generally unique since $\left\{\widehat{\boldsymbol{u}}_{i j}\right\}$ and $\left\{\widehat{\boldsymbol{v}}_{i j}\right\}$ can be chosen rather arbitrarily for $j \neq 1$. This further implies that the maximizer $\left\{\boldsymbol{Q}_{i}\right\}$ of $\mathcal{P}_{4}$ is also not unique, which is in agreement with the common understanding that rotation matrices are not necessarily unique when the dimension grows large.

\subsection{Grab-n-Pull (Optimization w. r. t. $\left.\left\{\lambda_{i}\right\}\right)$}

Note that, once the optimal $\left\{\boldsymbol{Q}_{i}\right\}$ is used, the objectives of $\mathcal{P}_{3}$ and $\mathcal{P}_{4}$ can be considered interchangeably. We assume that the optimal $\boldsymbol{w}$ and $\left\{\boldsymbol{Q}_{i}\right\}$ are obtained according to the above discussions, and are fixed. Therefore, to find $\left\{\lambda_{i}\right\}$, we can equivalently focus on obtaining the maximizer $\left\{\lambda_{i}\right\}$ of $\mathcal{P}_{3}$ via the optimization problem:

$$
\begin{aligned}
\Lambda: & \max _{\left\{\lambda_{i}\right\}} \min _{i \in[K]}\left\{\lambda_{i}\right\}-\eta \sum_{i=1}^{K}\left(\left\|\boldsymbol{A}_{i}^{\frac{1}{2}} \boldsymbol{w}\right\|_{2}-\sqrt{\lambda_{i}}\left\|\boldsymbol{B}_{i}^{\frac{1}{2}} \boldsymbol{w}\right\|_{2}\right)^{2} \\
& \text { s. t. } \quad \lambda_{i} \geq 0, \forall i \in[K] .
\end{aligned}
$$

Definition 1. Let $\left\{\lambda_{i}^{\star}\right\}$ denote the optimal $\left\{\lambda_{i}\right\}$ of $\Lambda$, and $\lambda^{\star} \triangleq$ $\min _{i \in[K]}\left\{\lambda_{i}^{\star}\right\}$. We let $\Upsilon$ to denote the set of all indices $m$ for which $\lambda_{m}^{\star}$ takes the minimal value among all $\left\{\lambda_{i}^{\star}\right\}$, i.e.

$$
\Upsilon=\left\{m \in[K]: \lambda_{m}^{\star}=\lambda^{\star}\right\}
$$

Moreover, we refer to $\gamma_{i}^{2} \triangleq\left\|\boldsymbol{A}_{i}^{\frac{1}{2}} \boldsymbol{w}\right\|_{2}^{2} /\left\|\boldsymbol{B}_{i}^{\frac{1}{2}} \boldsymbol{w}\right\|_{2}^{2}$ as the shadow value of $\lambda_{i}^{\star}$, for all $i \in[K]$.

It is straightforward to verify from the objective of $\Lambda$ that if $\lambda_{i}^{\star}>$ $\lambda^{\star}$, then $\lambda_{i}^{\star}=\gamma_{i}^{2}$. On the other hand, to obtain $\lambda^{\star}$, we need to maximize the criterion:

$$
f(\lambda)=\lambda-\eta \sum_{k \in \Upsilon}\left(\left\|\boldsymbol{A}_{k}^{\frac{1}{2}} \boldsymbol{w}\right\|_{2}-\sqrt{\lambda}\left\|\boldsymbol{B}_{k}^{\frac{1}{2}} \boldsymbol{w}\right\|_{2}\right)^{2} .
$$

Provided that $\eta$ is large enough (see the lower bound in Theorem 1), the optimal $\lambda^{\star}$ of the above quadratic criterion (in $\sqrt{\lambda}$ ) is given by

$$
\sqrt{\lambda^{\star}}=\frac{\eta \sum_{k \in \Upsilon} \alpha_{k} \beta_{k}}{\eta \sum_{k \in \Upsilon} \beta_{k}^{2}-1}
$$

in which $\alpha_{k} \triangleq\left\|\boldsymbol{A}_{k}^{\frac{1}{2}} \boldsymbol{w}\right\|_{2}$, and $\beta_{k} \triangleq\left\|\boldsymbol{B}_{k}^{\frac{1}{2}} \boldsymbol{w}\right\|_{2}$. It is interesting to have some insight into what $\sqrt{\lambda^{\star}}$ represents: Note that (24) can be rewritten as

$$
\sqrt{\lambda^{\star}}=\frac{\sum_{k \in \Upsilon} \gamma_{k} \beta_{k}^{2}}{\sum_{k \in \Upsilon} \beta_{k}^{2}-1 / \eta} .
$$

As a result, $\sqrt{\lambda^{\star}}$ can be viewed as a weighted average of $\gamma_{k}$ for $k \in \Upsilon$ - except that the term $-1 / \eta$ in the denominator of (25) makes $\sqrt{\lambda^{\star}} a$ bit larger than the actual weighted average. However, for an increasing $\eta, \sqrt{\lambda^{\star}}$ converges to the exact value of the weighted average specified above.

Hereafter, we propose a recursive Grab-n-Pull procedure to fully determine $\Upsilon$, while we can obtain $\sqrt{\lambda^{\star}}$ via (24). The proposed approach will make use of the following observation:

Lemma 2. If $\gamma_{i}^{2}<\lambda^{\star}$ for any $i \in[K]$, then $i \in \Upsilon$.

Proof. The inequality $\gamma_{i}^{2}<\lambda^{\star}$ implies that $\lambda_{i}^{\star} \neq \gamma_{i}^{2}$. Considering the discussion above (23), one can conclude that $\lambda_{i}^{\star} \leq \lambda^{\star}$, which due to the definition of $\lambda^{\star}$ yields $\lambda_{i}^{\star}=\lambda^{\star}$. Hence, the proof is complete.

Without loss of generality, and for the sake of simplicity, we assume in the sequel that the matrix pairs $\left\{\left(\boldsymbol{A}_{i}, \boldsymbol{B}_{i}\right)\right\}$ are sorted in such a way to form the ascending order:

$$
0 \leq \gamma_{1} \leq \gamma_{2} \leq \cdots \leq \gamma_{K}
$$

Based on the above ordering, the Grab-n-Pull approach is described in Table 1. Moreover, an illustration of the method is depicted in Fig. 1. The name of the method, i.e. Grab-n-Pull, comes from the intuition that the method grabs and pulls the lowest values of $\left\{\lambda_{i}\right\}$ to a level which is suitable for optimization of the alternative objectives, while achieving equality, at least for the lowest $\lambda_{i} \mathrm{~s}$. Due to the key role of Grab-n-Pull procedure in the proposed optimization framework, we also use the term Grab-n-Pull when referring to the general framework. In the following, we present a specific criterion on $\eta$ that not only bounds the objective of $\mathcal{P}_{4}$ (and $\mathcal{P}_{3}$ ), but also guarantees the convergence of the proposed approach.

Theorem 1. Suppose $\eta$ is chosen such that

$$
\eta>\eta_{l b} \triangleq \frac{1}{P}\left(\max _{i \in[K]}\left\{\sigma_{\min }^{-1}\left(\boldsymbol{B}_{k}\right)\right\}\right) .
$$

Then, $f(\lambda)$ is upper bounded (at its maximizer $\lambda^{\star}$, see (24)) as

$$
\begin{aligned}
f\left(\lambda^{\star}\right) & \leq \frac{\sum_{k \in \Upsilon} \alpha_{k}^{2}}{\sum_{k \in \Upsilon} \beta_{k}^{2}-1 / \eta} \\
& \leq \sigma_{\max }\left\{\left(\left(\sum_{k \in \Upsilon} \boldsymbol{B}_{k}\right)-\frac{1}{\eta P} \boldsymbol{I}\right)^{-1}\left(\sum_{k \in \Upsilon} \boldsymbol{A}_{k}\right)\right\} .
\end{aligned}
$$

The proof of Theorem 1 is omitted herein due to lack of space. Nevertheless, to see why the latter result implies the convergence of our algorithm, one can observe that different steps of the proposed framework lead to an increasing objective of $\mathcal{P}_{4}$ (and $\mathcal{P}_{3}$ ). To guarantee convergence in terms of the objective value, we only need to show that the objective is bounded from above-a condition which will be met by satisfying (27). 


\section{Table 1. Recursive Grab-n-Pull Procedure to Determine $\Upsilon$}

Step 0: Set $\Upsilon=\varnothing$.

Step 1: Include 1 in $\Upsilon$.

Remark: Based on Lemma 2, the primitive index 1 belongs to $\Upsilon$, as $\sqrt{\lambda^{\star}}$ is always larger than $\gamma_{1}$.

Step 2: Given the current index set of minimal variables $\Upsilon$, obtain $\sqrt{\lambda^{\star}}$ using (24).

Remark: Note that if $\sqrt{\lambda^{\star}}$ is smaller than $\gamma_{k}$ for all $k \in[K] \backslash \Upsilon$ then the obtained $\Upsilon$ is optimal, as all $\lambda_{k}$ with $k \in[K] \backslash \Upsilon$ have chosen their values freely to maximize the objective of $\Lambda$; as a result, adding other indices to $\Upsilon$ will lead to a decreased objective of $\Lambda$

Step 3: Let $\{h\} \subset[K]$ denote the indices for which $h \notin \Upsilon$. If $\gamma_{h} \leq$ $\sqrt{\lambda^{\star}}$, include $h$ in $\Upsilon$, and goto Step 2; otherwise stop.

Remark: This is a direct consequence of Lemma 2, particularly considering that $\sqrt{\lambda^{\star}}$ is only increasing with growing $|\Upsilon|$, which corresponds to adding larger $\gamma_{i}$ s to the weighted sum in (25).

\section{A NUMERICAL EXAMPLE WITH DISCUSSIONS}

In this section, a brief numerical example is provided to investigate the performance of the proposed method (performing the optimization w. r. t. to all variables at each iteration). To this end, we consider a downlink transmitter with $M=4$ antennas, as well as $K=4$ single-antenna users. The entries of the channel vectors $\boldsymbol{h}_{i}$ are drawn from an i.i.d. complex Gaussian distribution with zeromean and unit-variance. The Gaussian noise components received at each user antenna are assumed to have unit variance, i.e. $\sigma_{i}^{2}=1$ for all $i \in[K]$. We consider a total-power constraint with $P=1$, and stop the optimization iterations whenever the objective increase becomes bounded by $\epsilon=10^{-6}$.

Note that while the shadow values $\left\{\gamma_{i}^{2}\right\}$ represent the value of the fractional quadratic terms in the original objective $\mathcal{P}_{1}$, the auxiliary variables $\left\{\lambda_{i}\right\}$ tend to be as close as possible to $\left\{\gamma_{i}^{2}\right\}$ depending on the weight $(\eta)$ of the penalty-terms in $\mathcal{P}_{3}$ and $\mathcal{P}_{4}$. In Fig. 2, we present the transition of variables $\left\{\gamma_{i}^{2}\right\}$ and $\left\{\lambda_{i}\right\}$ vs. the iteration number for two different settings of $\eta$; namely $\eta=5$ and $\eta=100$. Although with a larger $\eta$ one may expect a lower value of the penalty functions in $\mathcal{P}_{3}$ and $\mathcal{P}_{4}$, a lower $\eta$ can play a useful role in speeding up the algorithm. Specifically, one can easily see that if $\eta$ is large, the value $\lambda^{\star}$ in (25) will be slightly greater than the weighted average of $\left\{\gamma_{k}^{2}\right\}_{k \in \Upsilon}$, whereas for smaller values of $\eta$, the bounces from the weighted average are much larger - and the convergence can occur much quicker. This phenomenon can also be observed in Fig. 2., noting that the aforementioned values of $\eta$ are chosen to accentuate the trade-off originated from the selection of $\eta$.

It should be mentioned that for the special case of the totalpower constraint, all optimal $\left\{\lambda_{i}\right\}$ are shown to be identical [1], i.e. $\lambda_{i}=\lambda^{\star}$ for all $i \in[K]$. This explains the identical values obtained by the method in Fig. 2 . The results leading to Fig. 2 were obtained in a few seconds on a standard PC.

\section{CONCLUSION}

An optimization framework for efficient precoding/beamforming in fairness-achieving networks was proposed. Thanks to a quadratic reformulation of the original problem, the proposed method can handle different signal constraints by employing the power method-like iterations. Various aspects of the proposed approach were studied.

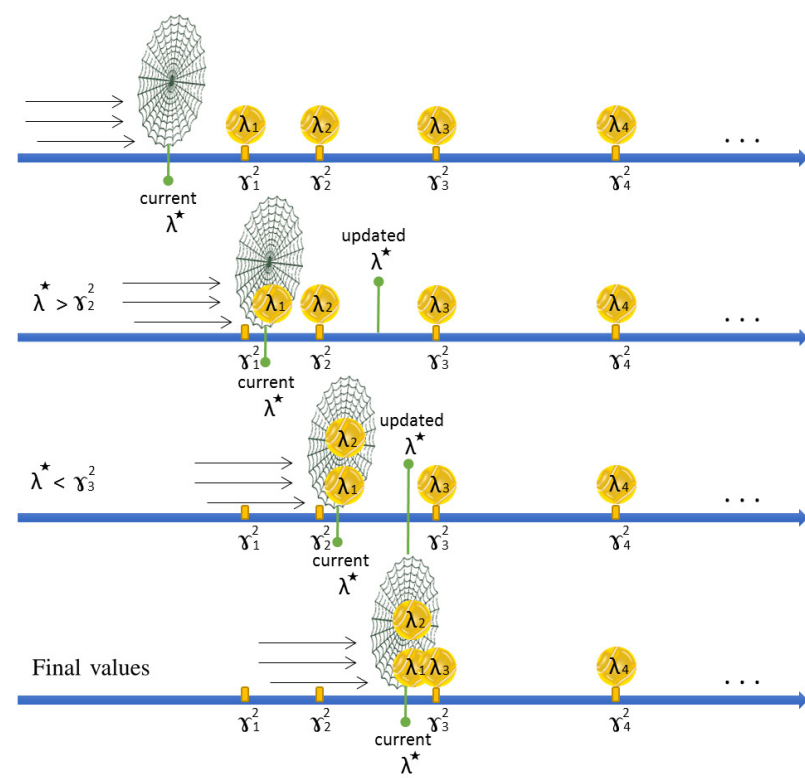

Fig. 1. An illustration of the Grab-n-Pull procedure. The optimal values of $\left\{\lambda_{i}\right\}$ are obtained when $\lambda^{\star}<\gamma_{3}^{2}$, which sets $\Upsilon$ to $\{1,2\}$.
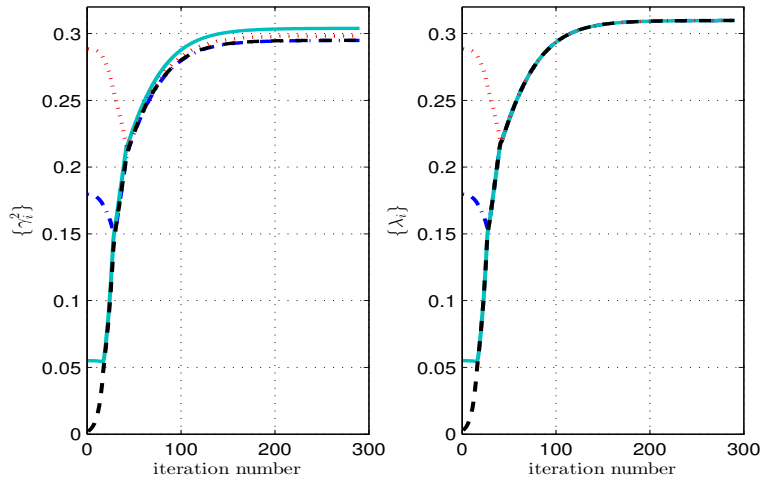

(a)
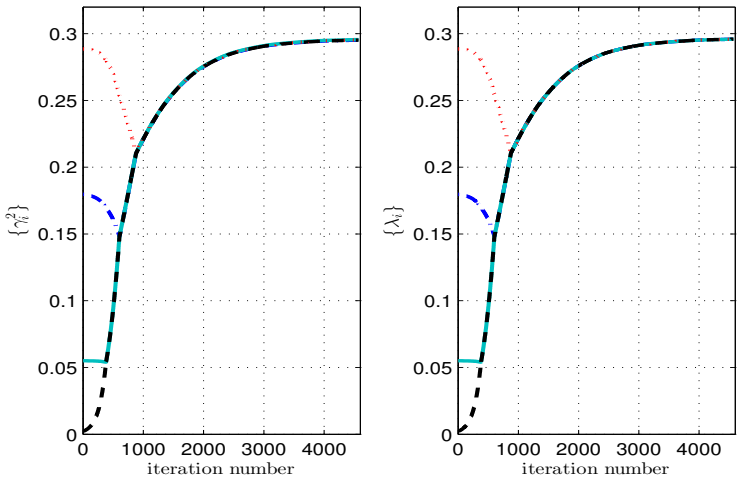

(b)

Fig. 2. Transition of the optimization parameters (distinguished by colors and line-styles) vs. the iteration number for different weights ( $\eta$ ) of the penalty-term in $\mathcal{P}_{3}$ and $\mathcal{P}_{4}$ : (a) $\eta=5$, and (b) $\eta=100$. 


\section{REFERENCES}

[1] M. Schubert and H. Boche, "Solution of the multiuser downlink beamforming problem with individual SINR constraints," IEEE Transactions on Vehicular Technology, vol. 53, no. 1, pp. 18-28, Jan. 2004.

[2] D. Cai, T. Quek, and C. W. Tan, "A unified analysis of max-min weighted SINR for MIMO downlink system," IEEE Transactions on Signal Processing, vol. 59, no. 8, pp. 3850-3862, Aug. 2011.

[3] A. Wiesel, Y. Eldar, and S. Shamai, "Linear precoding via conic optimization for fixed MIMO receivers," IEEE Transactions on Signal Processing, vol. 54, no. 1, pp. 161-176, Jan. 2006.

[4] Y.-F. Liu, Y.-H. Dai, and Z.-Q. Luo, "Max-min fairness linear transceiver design for a multi-user MIMO interference channel," IEEE Transactions on Signal Processing, vol. 61, no. 9, pp. 2413-2423, May 2013.

[5] C. W. Tan, M. Chiang, and R. Srikant, "Maximizing sum rate and minimizing MSE on multiuser downlink: Optimality, fast algorithms and equivalence via max-min SINR," IEEE Transactions on Signal Processing, vol. 59, no. 12, pp. 6127-6143, 2011.

[6] E. Karipidis, N. D. Sidiropoulos, and Z.-Q. Luo, "Quality of service and max-min fair transmit beamforming to multiple cochannel multicast groups," IEEE Transactions on Signal Processing, vol. 56, no. 3, pp. 1268-1279, 2008.

[7] D. W. Cai, T. Q. Quek, C. W. Tan, and S. H. Low, "Maxmin SINR coordinated multipoint downlink transmissionduality and algorithms," IEEE Transactions on Signal Processing, vol. 60, no. 10, pp. 5384-5395, 2012.

[8] G. Dartmann, X. Gong, W. Afzal, and G. Ascheid, "On the duality of the max-min beamforming problem with per-antenna and per-antenna-array power constraints," IEEE Transactions on Vehicular Technology, vol. 62, no. 2, pp. 606-619, Feb. 2013.

[9] M. Bengtsson and B. Ottersten, Optimal and Suboptimal Transmit Beamforming, ser. Electrical Engineering \& Applied Signal Processing Series, L. Chand Godara, Ed. CRC Press, 2001.

[10] M. M. Naghsh, M. Soltanalian, P. Stoica, M. ModarresHashemi, A. De Maio, and A. Aubry, "A Doppler robust design of transmit sequence and receive filter in the presence of signal-dependent interference," IEEE Transactions on Signal Processing, vol. 62, no. 4, pp. 772-785, 2014.

[11] A. Aubry, A. De Maio, M. Piezzo, M. M. Naghsh, M. Soltanalian, and P. Stoica, "Cognitive radar waveform design for spectral coexistence in signal-dependent interference," in IEEE Radar Conference. IEEE, 2014, pp. 474-478.

[12] A. Khabbazibasmenj, F. Roemer, S. Vorobyov, M. Haardt et al., "Sum-rate maximization in two-way AF MIMO relaying: Polynomial time solutions to a class of DC programming problems," IEEE Transactions on Signal Processing, vol. 60, no. 10, pp. 5478-5493, 2012.

[13] M. M. Naghsh, M. Soltanalian, P. Stoica, M. Masjedi, and B. Ottersten, "Efficient sum-rate maximization for massive MIMO AF-relay networks," IEEE Transactions on Wireless Communications, submitted, 2015.
[14] R. A. Horn and C. R. Johnson, Matrix Analysis, 2nd ed. New York, USA: Cambridge University Press, 2012.

[15] S. Zhang and Y. Huang, "Complex quadratic optimization and semidefinite programming," SIAM Journal on Optimization, vol. 16, no. 3, pp. 871-890, 2006.

[16] M. Soltanalian and P. Stoica, "Designing unimodular codes via quadratic optimization," IEEE Transactions on Signal Processing, vol. 62, no. 5, pp. 1221-1234, March 2014.

[17] M. Soltanalian, B. Tang, J. Li, and P. Stoica, "Joint design of the receive filter and transmit sequence for active sensing," IEEE Signal Processing Letters, vol. 20, no. 5, pp. 423-426, 2013.

[18] M. Soltanalian, H. Hu, and P. Stoica, "Single-stage transmit beamforming design for MIMO radar," Signal Processing, vol. 102, pp. 132-138, 2014.

[19] A. De Maio, Y. Huang, D. Palomar, S. Zhang, and A. Farina, "Fractional QCQP with applications in ML steering direction estimation for radar detection," IEEE Transactions on Signal Processing, vol. 59, no. 1, pp. 172-185, 2011.

[20] Z.-Q. Luo, W. K. Ma, A.-C. So, Y. Ye, and S. Zhang, "Semidefinite relaxation of quadratic optimization problems," IEEE Signal Processing Magazine, vol. 27, no. 3, pp. 20 -34, May 2010. 